\title{
ON A POWERED BOHR INEQUALITY
}

\author{
Ilgiz R. Kayumov and Saminathan Ponnusamy
}

\author{
Kazan Federal University \\ Kremlevskaya 18, 420008 Kazan, Russia; ikayumov@kpfu.ru \\ Indian Institute of Technology Madras, Department of Mathematics \\ Chennai-600 036, India; samy@isichennai.res.in, samy@iitm.ac.in
}

\begin{abstract}
The object of this paper is to study the powered Bohr radius $\rho_{p}, p \in(1,2)$, of analytic functions $f(z)=\sum_{k=0}^{\infty} a_{k} z^{k}$ defined on the unit disk $|z|<1$ and such that $|f(z)|<1$ for $|z|<1$. More precisely, if $M_{p}^{f}(r)=\sum_{k=0}^{\infty}\left|a_{k}\right|^{p} r^{k}$, then we show that $M_{p}^{f}(r) \leq 1$ for $r \leq r_{p}$ where $r_{\rho}$ is the powered Bohr radius for conformal automorphisms of the unit disk. This answers the open problem posed by Djakov and Ramanujan in 2000. A couple of other consequences of our approach is also stated, including an asymptotically sharp form of one of the results of Djakov and Ramanujan. In addition, we consider a similar problem for sense-preserving harmonic mappings in $|z|<1$. Finally, we conclude by stating the Bohr radius for the class of Bieberbach-Eilenberg functions.
\end{abstract}

\section{Preliminaries and main results}

Let $\mathcal{B}$ denote the class of analytic functions $f$ defined on the unit disk $\mathbf{D}:=$ $\{z \in \mathbf{C}:|z|<1\}$, with the power series expansion $f(z)=\sum_{k=0}^{\infty} a_{k} z^{k}$ and such that $|f(z)|<1$ for $z \in \mathbf{D}$. Then the classical Bohr's inequality states that there is a constant $\rho$ such that

$$
M^{f}(r):=\sum_{k=0}^{\infty}\left|a_{k}\right| r^{k} \leq 1 \text { for all } r=|z| \leq \rho
$$

and the value $\rho=1 / 3$ is optimal. The number $\rho=1 / 3$, known as Bohr's radius, was originally obtained in 1914 by Bohr [6] with $\rho=1 / 6$, but subsequently later, Wiener, Riesz and Schur, independently established the sharp inequality for $r=|z| \leq 1 / 3$. This little article of Bohr generates intensive research activities even after a century of its appearance. We refer to the recent survey article on this topic [4] and the references therein. Multidimensional generalizations of this result were obtained by Boas and Khavinson [5] by establishing upper and lower bounds for the Bohr radius of the unit polydisk $\mathbf{D}^{n}$. Aizenberg $[2,3]$ extended the concept of Bohr radius in several different directions for further studies in this topic. In 2000, Djakov and Ramanujan [10] investigated the same phenomenon from different point of view. For $f \in \mathcal{B}$ and a fixed $p>0$, we consider the powered Bohr sum $M_{p}^{f}(r)$ defined by

$$
M_{p}^{f}(r)=\sum_{k=0}^{\infty}\left|a_{k}\right|^{p} r^{k} .
$$

https://doi.org/10.5186/aasfm.2019.4416

2010 Mathematics Subject Classification: Primary 30A10, 30H05, 30C35; Secondary 30C45.

Key words: Bounded analytic functions, $p$-symmetric functions, Bohr's inequality, subordination, harmonic mappings, Bieberbach-Eilenberg functions. 
Observe that for $p=1, M_{p}^{f}(r)$ reduces to the classical Bohr sum defined as above by $M^{f}(r)$. The best possible constant $\rho_{p}$ for which

$$
M_{p}^{f}(r) \leq 1 \text { for all } r \leq \rho_{p}
$$

is called the (powered) Bohr radius for the family $\mathcal{B}$.

We now introduce

$$
M_{p}(r):=\sup _{f \in \mathcal{B}} M_{p}^{f}(r)
$$

and

$$
r_{p}:=\sup \left\{r: a^{p}+\frac{r\left(1-a^{2}\right)^{p}}{1-r a^{p}} \leq 1,0 \leq a<1\right\}=\inf _{a \in[0,1)} \frac{1-a^{p}}{a^{p}\left(1-a^{p}\right)+\left(1-a^{2}\right)^{p}} .
$$

Let us first proceed to recall the following results.

Theorem A. [10, Theorem 3] For each $p \in(1,2]$ and $f(z)=\sum_{k=0}^{\infty} a_{k} z^{k}$ belongs to $\mathcal{B}$, we have $M_{p}^{f}(r) \leq 1$ for $r \leq T_{p}$, where

$$
m_{p} \leq T_{p} \leq r_{p}
$$

Here $r_{p}$ is as above,

$$
m_{p}:=\frac{p}{\left(2^{1 /(2-p)}+p^{1 /(2-p)}\right)^{2-p}} \text { for } 1<p<2,
$$

and $m_{2}:=\lim _{p \rightarrow 2} m_{p}=1$.

Theorem B. [10, Theorem 2] For each $p \in(0,2)$,

$$
M_{p}(r) \asymp\left(\frac{1}{1-r}\right)^{1-p / 2} .
$$

Our first aim is to investigate the problem posed by Djakov and Ramanujan [10] about the Bohr radius for $M_{p}^{f}(r)$. Their question is the following.

Problem 1. [10, Question 1, p. 71] What is the exact value of the (powered) Bohr radius $\rho_{p}, p \in(1,2)$ ? Is it true that $\rho_{p}=r_{p}$ ?

Using the method of proofs of our recent approach from [12, 13], we solve this problem affirmatively in the following form.

Theorem 1. If $f(z)=\sum_{k=0}^{\infty} a_{k} z^{k}$ belongs to $\mathcal{B}$ and $0<p \leq 2$, then

$$
M_{p}(r)=\max _{a \in[0,1]}\left\{a^{p}+\frac{r\left(1-a^{2}\right)^{p}}{1-r a^{p}}\right\}, \quad 0 \leq r \leq 2^{p / 2-1},
$$

and

$$
M_{p}(r)<\left(\frac{1}{1-r^{2 /(2-p)}}\right)^{1-p / 2}, \quad 2^{p / 2-1}<r<1 .
$$

Proofs of Theorem 1 and a couple of its corollaries will be given in Section 2 .

Let us remark that $M_{p}(r)=1$ for $p \geq 2$ and $r<1$. So, the interesting case is to consider the problem only for $p \in(1,2)$.

One may ask about the second inequality of Theorem 1: how close it to be sharp? To get an answer to this question we will use a Bombieri-Bourgain estimate [8] which reads as follows: for a given $\varepsilon>0$, there exists a positive constant $C(\varepsilon)>0$, such that

$$
M_{1}(\rho) \geq \frac{1}{\sqrt{1-\rho^{2}}}-C(\varepsilon)\left(\log \frac{1}{1-\rho}\right)^{(3 / 2)+\varepsilon}, \quad \rho \geq 1 / \sqrt{2} .
$$


The Hölder inequality implies that

$$
\begin{aligned}
M_{1}^{f}\left(r^{1 /(2-p)}\right) & =\sum_{k=0}^{\infty}\left|a_{k}\right| r^{k / p} r^{(2 k(p-1)) /(p(2-p))} \\
& \leq\left(\sum_{k=0}^{\infty}\left|a_{k}\right|^{p} r^{k}\right)^{1 / p}\left(\sum_{k=0}^{\infty} r^{2 k /(2-p)}\right)^{1-1 / p} \\
& =\left(M_{p}^{f}(r)\right)^{1 / p} \frac{1}{\left(1-r^{2 /(2-p)}\right)^{(p-1) / p}}
\end{aligned}
$$

so that

$$
M_{p}^{f}(r) \geq\left(\frac{1}{\sqrt{1-r^{2 /(2-p)}}}-C(\varepsilon)\left(\log \frac{1}{1-r^{1 /(2-p)}}\right)^{3 / 2+\varepsilon}\right)^{p}\left(1-r^{2 /(2-p)}\right)^{p-1}
$$

for $2^{p / 2-1}<r<1$; or equivalently

$$
M_{p}^{f}(r) \geq\left(\frac{1}{1-r^{2 /(2-p)}}\right)^{1-p / 2}-C_{1}(\varepsilon)\left(1-r^{2 /(2-p)}\right)^{(p-1) / 2}\left(\log \frac{1}{1-r^{1 /(2-p)}}\right)^{3 / 2+\varepsilon} .
$$

This estimate together with the second estimate of Theorem 1 implies that

$$
M_{p}(r)-\left(\frac{1}{1-r^{2 /(2-p)}}\right)^{1-p / 2} \rightarrow 0 \text { as } r \rightarrow 1^{-}
$$

for $1<p<2$ while we do not know whether this fact is true for $p=1$. Also the last estimate can be considered as an asymptotically sharp form of Theorem B in the case $p>1$.

Corollary 1. Let $p \in(1,2)$. Then $M_{p}(r)=1$ for $r \leq r_{p}$.

In [16, Corollary 2.8], Paulsen et al. showed that if $f \in \mathcal{B}$, then for $r \in[0,1)$,

$$
M_{1}^{f}(r) \leq m(r)=\inf \left\{M(r), 1 / \sqrt{1-r^{2}}\right\}
$$

where

$$
M(r)=\sup \left\{t+\left(1-t^{2}\right) \frac{r}{1-r}: 0 \leq t \leq 1\right\}= \begin{cases}1 & \text { for } 0 \leq r \leq 1 / 3 \\ \frac{4 r^{2}+(1-r)^{2}}{4 r(1-r)} & \text { for } 1 / 3<r<1 .\end{cases}
$$

In 2002, Paulsen et al. [16] raised a question whether the inequality (1) is sharp for any $r$ with $1 / 3<r<1$. However, in 1962 this has been answered by Bombieri [7] who determined the exact value of this constant for $r$ in the range $1 / 3 \leq r \leq 1 / \sqrt{2}$. This constant is

$$
m(r)=\frac{3-\sqrt{8\left(1-r^{2}\right)}}{r} .
$$

Further results on this and related topics can be found in $[10,16]$. On the other hand, it is worth mentioning that the answer to the above question is indeed a consequence of Theorem 1 and so, we state it as a corollary.

Corollary 2. We have the following sharp estimate:

$$
M_{1}(r)=\frac{1}{r}\left(3-\sqrt{8\left(1-r^{2}\right)}\right) \text { for } r \in\left[\frac{1}{3}, \frac{1}{\sqrt{2}}\right] .
$$

Finally, we recall the following corollary which was proved in [13] and so we omit the proof. 
Corollary 3. Let $p \in \mathbf{N}$ and $0 \leq m \leq p, f(z)=\sum_{k=0}^{\infty} a_{p k+m} z^{p k+m}$ be analytic in $\mathbf{D}$ and $|f(z)|<1$ in $\mathbf{D}$. Then

$$
\sum_{k=0}^{\infty}\left|a_{p k+m}\right| r^{p k+m} \leq 1 \text { for } r \leq r_{p, m}
$$

where $r_{p, m}$ is the maximal positive root of the equation

$$
-6 r^{p-m}+r^{2(p-m)}+8 r^{2 p}+1=0 .
$$

The extremal function has the form $z^{m}\left(z^{p}-a\right) /\left(1-a z^{p}\right)$, where

$$
a=\left(1-\frac{\sqrt{1-r_{p, m^{2 p}}}}{\sqrt{2}}\right) \frac{1}{r_{p, m}^{p}} .
$$

Our next result concerns sense-preserving harmonic mappings defined on the unit disk. Recall that the family $\mathcal{H}$ of complex-valued harmonic functions $f=h+\bar{g}$ defined on the unit disk $\mathbf{D}$ and its univalent subfamilies are investigated in details. Here $h$ and $g$ are analytic on $\mathbf{D}$ with the form

$$
h(z)=\sum_{k=0}^{\infty} a_{k} z^{k} \quad \text { and } \quad g(z)=\sum_{k=1}^{\infty} b_{k} z^{k}
$$

so that the Jacobian of $f$ is given by $J_{f}=\left|f_{z}\right|^{2}-\left|f_{\bar{z}}\right|^{2}=\left|h^{\prime}\right|^{2}-\left|g^{\prime}\right|^{2}$. We say that the locally univalent harmonic mapping $f$ is sense-preserving if $J_{f}(z)>0$ in $\mathbf{D}$. We call $\omega(z)=g^{\prime}(z) / h^{\prime}(z)$ the complex dilatation of $f=h+\bar{g}$. Lewy's theorem implies that every harmonic function $f$ on $\mathbf{D}$ is locally one-to-one and sense-preserving on $\mathbf{D}$ if and only if $|\omega(z)|<1$ for $z \in \mathbf{D}$. See [9, 11] for detailed discussion on the class of univalent harmonic mappings and its geometric subclasses.

Theorem 2. Suppose that $f(z)=h(z)+\overline{g(z)}=\sum_{k=0}^{\infty} a_{k} z^{k}+\overline{\sum_{k=1}^{\infty} b_{k} z^{k}}$ is a harmonic mapping of the disk $\mathbf{D}$, where $h$ is a bounded function in $\mathbf{D}$ and $\left|g^{\prime}(z)\right| \leq$ $\left|h^{\prime}(z)\right|$ for $z \in \mathbf{D}$ (the later condition obviously holds if $f$ is sense-preserving). If $p \in[0,2]$ then the following sharp inequality holds

$$
\left|a_{0}\right|^{p}+\sum_{k=1}^{\infty}\left(\left|a_{k}\right|^{p}+\left|b_{k}\right|^{p}\right) r^{k} \leq\|h\|_{\infty} \max _{a \in[0,1]}\left\{a^{p}+\frac{2 r\left(1-a^{2}\right)^{p}}{1-r a^{p}}\right\}
$$

for $r \leq\left(2^{1 /(p-2)}+1\right)^{p / 2-1}$. In the case $p>2$ we have

$$
\left|a_{0}\right|^{p}+\sum_{k=1}^{\infty}\left(\left|a_{k}\right|^{p}+\left|b_{k}\right|^{p}\right) r^{k} \leq\|h\|_{\infty} \max \{1,2 r\} .
$$

Corollary 4. Suppose that $f(z)=h(z)+\overline{g(z)}=\sum_{k=0}^{\infty} a_{k} z^{k}+\overline{\sum_{k=1}^{\infty} b_{k} z^{k}}$ is a sense-preserving harmonic mapping of the disk $\mathbf{D}$, where $h$ is a bounded function in D. Then the following sharp inequalities holds:

$$
\left|a_{0}\right|+\sum_{k=1}^{\infty}\left(\left|a_{k}\right|+\left|b_{k}\right|\right) r^{k} \leq \frac{\|h\|_{\infty}}{r}\left(5-2 \sqrt{6} \sqrt{1-r^{2}}\right) \text { for } \frac{1}{5} \leq r \leq \sqrt{\frac{2}{3}},
$$

and

$$
\left|a_{0}\right|+\sum_{k=1}^{\infty}\left(\left|a_{k}\right|+\left|b_{k}\right|\right) r^{k} \leq\|h\|_{\infty} \text { for } r \leq \frac{1}{5}
$$

Proofs of Theorem 2 and Corollary 4 will be given in Section 2. In Section 3, we discuss Bohr radius for the class of Bieberbach-Eilenberg functions. 


\section{Proofs of Theorems 1 and 2 and their corollaries}

The proofs of the theorems rely on a couple of lemmas established by the present authors in [12] (see also [13]).

Lemma 1. [12] Let $|a|<1$ and $0<R \leq 1$. If $g(z)=\sum_{k=0}^{\infty} b_{k} z^{k}$ belongs to $\mathcal{B}$, then the following sharp inequality holds:

$$
\sum_{k=1}^{\infty}\left|b_{k}\right|^{2} R^{k} \leq R \frac{\left(1-\left|b_{0}\right|^{2}\right)^{2}}{1-\left|b_{0}\right|^{2} R} .
$$

Lemma 2. For all $p \in(0,2)$, we have $r_{p}<(1 / 2)^{1-p / 2}=R_{p}$, where $r_{p}$ is defined as in the beginning.

Proof. Let $r=R_{p}$ and set $a=(1 / 2)^{1-p / 2}$. Then we conclude that

$$
a^{p}+r \frac{\left(1-a^{2}\right)^{p}}{1-r a^{p}}=2\left(\frac{1}{2}\right)^{p / 2}>1
$$

which contradicts to the definition of $r_{p}$.

Proof of Theorem 1. Let $\left|a_{0}\right|=a>0$ and $r \leq 2^{p / 2-1}$. At first we suppose that $a>r^{1 /(2-p)}$. In this case we have

$$
\begin{aligned}
M_{p}^{f}(r) & =a^{p}+\sum_{k=1}^{\infty} \rho^{k}\left|a_{k}\right|^{p}\left(\frac{r}{\rho}\right)^{k} \\
& \leq a^{p}+\left(\sum_{k=1}^{\infty}\left(\rho^{k}\left|a_{k}\right|^{p}\right)^{2 / p}\right)^{p / 2}\left(\sum_{k=1}^{\infty}\left(\frac{r}{\rho}\right)^{2 k /(2-p)}\right)^{1-p / 2} \\
& =a^{p}+\left(\sum_{k=1}^{\infty}\left(\rho^{2 / p}\right)^{k}\left|a_{k}\right|^{2}\right)^{p / 2}\left(\sum_{k=1}^{\infty}\left(\left(\frac{r}{\rho}\right)^{2 /(2-p)}\right)^{k}\right)^{(2-p) / 2} \\
& \leq a^{p}+\left(\frac{\rho^{2 / p}\left(1-a^{2}\right)^{2}}{1-a^{2} \rho^{2 / p}}\right)^{p / 2}\left(\frac{(r / \rho)^{2 /(2-p)}}{1-(r / \rho)^{2 /(2-p)}}\right)^{(2-p) / 2} \quad \text { (by Lemma 1) } \\
& =a^{p}+r\left(\frac{\left(1-a^{2}\right)^{2}}{1-a^{2} \rho^{2 / p}}\right)^{p / 2}\left(\frac{1}{1-(r / \rho)^{2 /(2-p)}}\right)^{(2-p) / 2} .
\end{aligned}
$$

Setting $\rho=r^{p / 2} a^{(p-2) p / 2}$ we obtain the inequality

$$
M_{p}^{f}(r) \leq a^{p}+r \frac{\left(1-a^{2}\right)^{p}}{1-r a^{p}}
$$

which proves the theorem in the case $a>r^{1 /(2-p)}$.

In the case $a \leq r^{1 /(2-p)}$, we set $\rho=1$ and obtain

$$
M_{p}^{f}(r)=\sum_{k=0}^{\infty}\left|a_{k}\right|^{p} r^{k} \leq a^{p}+r \frac{\left(1-a^{2}\right)^{p / 2}}{\left(1-r^{2 /(2-p)}\right)^{1-p / 2}} .
$$

Let us remark that the inequality $M_{p}^{f}(r) \leq 1$ is valid in the cases $a=0$ and $a=$ $r^{1 /(2-p)}$. This fact can be established as a limiting case of the previous case. Finally, we let $t=a^{2}$. We have then to maximize the expression

$$
A(t)=t^{p / 2}+r \frac{(1-t)^{p / 2}}{\left(1-r^{2 /(2-p)}\right)^{1-p / 2}}, \quad t \leq r^{2 /(2-p)} .
$$


Using differentiation we obtain the stationary point

$$
t=1-r^{2 /(2-p)}
$$

which must satisfy under the restriction $t \leq r^{2 /(2-p)}$ which is impossible because $r \leq 2^{p / 2-1}$.

However, in the case $r>2^{p / 2-1}$ the critical point $t$ is admissible so that

$$
A(t)=t^{p / 2}+r \frac{(1-t)^{p / 2}}{\left(1-r^{2 /(2-p)}\right)^{1-p / 2}}=\left(\frac{1}{1-r^{2 /(2-p)}}\right)^{1-p / 2} .
$$

This observation shows that

$$
M_{p}^{f}(r) \leq\left(\frac{1}{1-r^{2 /(2-p)}}\right)^{1-p / 2}, \quad 2^{p / 2-1}<r<1 .
$$

Now let us show that this inequality cannot be sharp. To do this we will use the method presented by Bombieri and Bourgain [8].

Suppose that the estimate sharp in this case. Then by analyzing Hölder's inequality we immediately conclude that

$$
\left|a_{k}\right|=\sqrt{1-r^{2 /(2-p)}} r^{k /(2-p)}, \quad k \geq 0 .
$$

Also it is easy to show that the extremal function must be a Blashke product with a finite degree $d \geq 1$. Computing the area, one obtains that

$$
\pi d=\operatorname{Area} f(\mathbf{D})=\pi \sum_{k=1}^{\infty} k\left|a_{k}\right|^{2}=\pi \frac{\lambda^{2}}{1-\lambda^{2}}, \quad \lambda=r^{1 /(2-p)} .
$$

From here we easily deduce that $d=\lambda^{2} /\left(1-\lambda^{2}\right)$ and thus, $\lambda=\sqrt{d /(d+1))}$, which gives

$$
\sqrt{\frac{d}{d+1}}=r^{1 /(2-p)}, \text { i.e. } r=\left(\frac{d}{d+1}\right)^{1-(p / 2)} .
$$

Therefore our inequality could be sharp for these values only. Now let us show that this is possible for $d=1$ only. Using the same reasoning as in [8] (in fact we apply their considerations in which $r$ is replaced by $\left.r^{1 /(2-p)}\right)$ we arrive at the identity

$$
\sqrt{1-r^{2 /(2-p)}}=r^{d /(2-p)}
$$

which together with (2) implies that

$$
\sqrt{1-\frac{d}{d+1}}=\left(\frac{d}{d+1}\right)^{d / 2}
$$

which is equivalent to the equality

$$
\frac{1}{d+1}=\left(\frac{d}{d+1}\right)^{d}
$$

From classical analysis we know that the right hand side of this equality is greater than $1 / e$ for $d \geq 1$ so that $d+1 \leq e$ and from here we easily deduce that $d=1$ which concludes the proof of Theorem 1.

Proof of Corollary 1. Easily follows from Theorem 1 and Lemma 2.

Proof of Corollary 2. Theorem 1 for $p=1$ gives that

$$
M_{p}(r)=\max _{a \in[0,1]}\left\{a+\frac{r\left(1-a^{2}\right)}{1-r a}\right\} .
$$


By using differentiation it is easy to show that in the case $1 / 3 \leq r \leq 1 / \sqrt{2}$ the maximum of the last expression is achieved at the point

$$
a=\left(1-\frac{\sqrt{1-r^{2}}}{\sqrt{2}}\right) \frac{1}{r}
$$

and consequently, we obtain that

$$
M_{1}(r)=\frac{1}{r}\left(3-2 \sqrt{2} \sqrt{1-r^{2}}\right) .
$$

The proof is complete.

Proof of Theorem 2. Without lost of generality we may assume that $\|h\|_{\infty}=1$. As in [14], the condition $\left|g^{\prime}(z)\right| \leq\left|h^{\prime}(z)\right|$ gives that for each $r \in[0,1)$,

$$
\sum_{k=1}^{\infty}\left|b_{k}\right|^{2} r^{k} \leq \sum_{k=1}^{\infty}\left|a_{k}\right|^{2} r^{k}
$$

Let $\left|a_{0}\right|=a>0$. Then, by using the same method as in the previous theorem in the case $a>r^{1 /(2-p)}$, we obtain

$$
\left|a_{0}\right|^{p}+\sum_{k=1}^{\infty}\left(\left|a_{k}\right|^{p}+\left|b_{k}\right|^{p}\right) r^{k} \leq a^{p}+2 r \frac{\left(1-a^{2}\right)^{p}}{1-r a^{p}} .
$$

In the case $a \leq r^{1 /(2-p)}$, we let $\rho=1$ and obtain

$$
\sum_{k=0}^{\infty}\left|a_{k}\right|^{p} r^{k} \leq a^{p}+2 r \frac{\left(1-a^{2}\right)^{p / 2}}{\left(1-r^{2 /(2-p)}\right)^{1-p / 2}} .
$$

We set $t=a^{2}$. We have to maximize the expression

$$
B(t)=t^{p / 2}+2 r \frac{(1-t)^{p / 2}}{\left(1-r^{2 /(2-p)}\right)^{1-p / 2}}, \quad t \leq r^{2 /(2-p)} .
$$

Using differentiation we see that the function $B(t)$ is increasing on the interval

$$
0 \leq t \leq \frac{1-r^{2 /(2-p)}}{1+(2 r)^{2 /(2-p)}-r^{2 /(2-p)}}
$$

The upper bound of this interval is greater than or equal to $2^{p / 2-1}$ in the case $r \leq$ $\left(2^{1 /(p-2)}+1\right)^{p / 2-1}$. It means that the function $B(t)$ has maximum at the point $t=r^{2 /(2-p)}$ which corresponds to the case $a=r^{1 /(2-p)}$ so that we can apply our previous case. This completes the proof of Theorem 2 .

Let $p=1$ and then we apply the previous theorem. As a result, we obtain the inequality

$$
\left|a_{0}\right|+\sum_{k=1}^{\infty}\left(\left|a_{k}\right|+\left|b_{k}\right|\right) r^{k} \leq \max _{a \in[0,1]}\left\{a+\frac{2 r\left(1-a^{2}\right)}{1-r a}\right\} \text { for } r \leq \sqrt{2 / 3} .
$$

Straightforward calculations confirm the proof of Corollary 4. 


\section{Concluding remarks}

Let $\mathcal{B E}$ denote the class of all functions $f(z)=\sum_{k=1}^{\infty} a_{k} z^{k}$ analytic in $\mathbf{D}$ such that $f\left(z_{1}\right) f\left(z_{2}\right) \neq 1$ for all pairs of points $z_{1}, z_{2}$ in D. Each $f \in \mathcal{B E}$ is called a BieberbachEilenberg function. Clearly, $\mathcal{B E}$ contains the class $\mathcal{B}_{0}$, where $\mathcal{B}_{0}=\{f \in \mathcal{B}: f(0)=0\}$. In 1970, Aharonov [1] and Nehari [15] independently showed that

$$
\sum_{k=1}^{\infty}\left|a_{k}\right|^{2} \leq 1 \quad \text { and } \quad|f(z)| \leq \frac{|z|}{\sqrt{1-|z|^{2}}}
$$

hold for every $f \in \mathcal{B E}$. Equality holds only for the functions

$$
f(z)=\frac{\eta z}{R \pm\left(\sqrt{R^{2}-1}\right) i \eta z}, \quad R>1,|\eta|=1 .
$$

Since $\mathcal{B}_{0} \subset \mathcal{B E}$, it is natural to ask for the Bohr radius for the family $\mathcal{B E}$. Indeed, we see blow that the Bohr radius for $\mathcal{B E}$ and the class $\mathcal{B}_{0}$ remains the same.

Theorem 3. Assume that $f(z)=\sum_{k=1}^{\infty} a_{k} z^{k}$ belongs to $\mathcal{B E}$. Then

$$
\sum_{k=1}^{\infty}\left|a_{k}\right| r^{k} \leq 1 \text { for }|z|=r \leq 1 / \sqrt{2}
$$

The number $1 / \sqrt{2}$ is sharp.

Proof. Because $f \in \mathcal{B E}$ satisfies the coefficient inequality (4), it follows that

$$
\sum_{k=1}^{\infty}\left|a_{k}\right| r^{k} \leq \sqrt{\sum_{k=1}^{\infty}\left|a_{k}\right|^{2}} \sqrt{\sum_{k=1}^{\infty} r^{2 k}} \leq \frac{r}{\sqrt{1-r^{2}}}
$$

which is less than or equal to 1 if $0 \leq r \leq 1 / \sqrt{2}$. The number $1 / \sqrt{2}$ is sharp as the function $f(z)=z(a-z) /(1-a z)$ shows, where $a=1 / \sqrt{2}$. The proof is complete.

Theorem 4. Suppose that $f(z)=h(z)+\overline{g(z)}=\sum_{k=1}^{\infty} a_{k} z^{k}+\overline{\sum_{k=1}^{\infty} b_{k} z^{k}}$ is a harmonic mapping of the disk $\mathbf{D}$, where $h \in \mathcal{B E}$ and $\left|g^{\prime}(z)\right| \leq\left|h^{\prime}(z)\right|$ for $z \in \mathbf{D}$. Then for any $p \geq 1$ and $r<1$, the following inequality holds:

$$
\sum_{k=1}^{\infty}\left(\left|a_{k}\right|^{p}+\left|b_{k}\right|^{p}\right)^{1 / p} r^{k} \leq \max \left\{2^{(1 / p)-1 / 2}, 1\right\} \frac{\sqrt{2} r}{\sqrt{1-r^{2}}} .
$$

Proof. By hypothesis, (3) holds and thus, letting $r$ approach 1, we get

$$
\sum_{k=1}^{\infty}\left|b_{k}\right|^{2} \leq \sum_{k=1}^{\infty}\left|a_{k}\right|^{2} \leq 1
$$

Consequently, we obtain

$$
\begin{aligned}
\sum_{k=1}^{\infty}\left(\left|a_{k}\right|^{p}+\left|b_{k}\right|^{p}\right)^{1 / p} r^{k} & \leq \sqrt{\sum_{k=1}^{\infty}\left(\left|a_{k}\right|^{p}+\left|b_{k}\right|^{p}\right)^{2 / p} \sqrt{\sum_{k=1}^{\infty} r^{2 k}}} \\
& \leq \sqrt{\max \left\{2^{(2 / p)-1}, 1\right\} \sum_{k=1}^{\infty}\left(\left|a_{k}\right|^{2}+\left|b_{k}\right|^{2}\right) \frac{r}{\sqrt{1-r^{2}}}} \\
& \leq \max \left\{2^{(1 / p)-1 / 2}, 1\right\} \frac{\sqrt{2} r}{\sqrt{1-r^{2}}}
\end{aligned}
$$


and the proof is complete.

Theorem 4 for $p=1$ shows that for $r \leq 1 / \sqrt{5}$,

$$
\sum_{k=1}^{\infty}\left(\left|a_{k}\right|+\left|b_{k}\right|\right) r^{k} \leq 1 .
$$

Similarly, for $p=2$, we see that for $r \leq 1 / \sqrt{3}$,

$$
\sum_{k=1}^{\infty}\left(\left|a_{k}\right|^{2}+\left|b_{k}\right|^{2}\right)^{1 / 2} r^{k} \leq 1 .
$$

Acknowledgements. The research of the first author was funded by the subsidy allocated to Kazan Federal University for the state assignment in the sphere of scientific activities, project No. 1.12878.2018/12.1. The work of the second author is supported in part by Mathematical Research Impact Centric Support (MATRICS) grant, File No.: MTR/2017/000367, by the Science and Engineering Research Board (SERB), Department of Science and Technology (DST), Government of India.

\section{References}

[1] Aharonov, D.: On Bieberbach-Eilenberg functions. - Bull. Amer. Math. Soc. 76:1, 1970, $101-104$.

[2] Aizenberg, L.: Multidimensional analogues of Bohr's theorem on power series. - Proc. Amer. Math. Soc. 128:4, 2000, 1147-1155.

[3] AizenberG, L.: Generalization of Carathéodory's inequality and the Bohr radius for multidimensional power series. - In: Selected topics in complex analysis, Oper. Theory Adv. Appl. 158, 2005, 87-99.

[4] Ali, R. M., Y. Abu-Muhanna, and S. Ponnusamy: On the Bohr inequality. - In: Progress

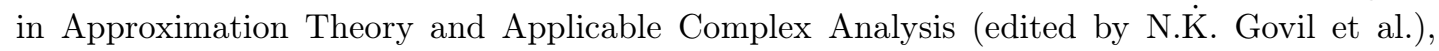
Springer Optim. Appl. 117, 2016, 265-295.

[5] Boas, H. P., and D. Khavinson: Bohr's power series theorem in several variables. - Proc. Amer. Math. Soc. 125:10, 1997, 2975-2979.

[6] Bohr, H.: A theorem concerning power series. - Proc. London Math. Soc. 13:2, 1914, 1-5.

[7] Bombieri, E.: Sopra un teorema di H. Bohr e G. Ricci sulle funzioni maggioranti delle serie di potenze. - Boll. Unione Mat. Ital. 17, 1962, 276-282.

[8] Bombieri, E., and J. Bourgain: A remark on Bohr's inequality. - Int. Math. Res. Not. IMRN $80,2004,4307-4330$.

[9] Clunie, J. G., and T. Sheil-Small: Harmonic univalent functions. - Ann. Acad. Sci. Fenn. Ser. A I Math. 9, 1984, 3-25.

[10] Djakov, P. B., and M. S. Ramanujan: A remark on Bohr's theorems and its generalizations. - J. Anal. 8, 2000, 65-77.

[11] Duren, P. L.: Harmonic mappings in the plane. - Cambridge Univ. Press, 2004.

[12] Kayumov, I. R., and S. Ponnusamy: Bohr inequality for odd analytic functions. - Comput. Methods Funct. Theory 17, 2017, 679-688.

[13] Kayumov, I. R., and S. Ponnusamy: Bohr's inequalities for the analytic functions with lacunary series and harmonic functions. - J. Math. Anal. Appl. 465, 2018, 857-871.

[14] Kayumov, I. R., S. Ponnusamy, and N. Shakirov: Bohr radius for locally univalent harmonic mappings. - Math. Nachr. 291, 2018, 1757-1768.

[15] Nehari, Z.: On the coefficients of Bieberbach-Eilenberg functions, - J. Anal. Math. 23, 1970, $297-303$. 
[16] Paulsen, V.I., G. Popescu, and D. Singh: On Bohr's inequality. - Proc. London Math. Soc. 85:2, 2002, 493-512.

Received 11 April $2017 \bullet$ Accepted 30 August 2018 